\title{
Freedom in nature
}

\section{P. Hájíček}

Received: 4 March 2009 / Accepted: 29 May 2009 / Published online: 20 June 2009

(C) Springer Science+Business Media, LLC 2009

\begin{abstract}
The paper starts with the proposal that the cause of the apparent insolubility of the free-will problem are several popular but strongly metaphysical notions and hypotheses. To reduce the metaphysics, some ideas are borrowed from physics. A concept of event causality is discussed. The importance of Hume's Principle of Causality is stressed and his Principle of Causation is weakened. The key concept of the paper, the so-called relative freedom, is also suggested by physics. It is a kind of freedom that can be observed everywhere in nature. Turning to biology, incomplete knowledge is defined for all organisms. They cope with the problem by Popper's trial and error processes. One source of their success is the relative freedom of choice from the basic option ranges: mutations, motions and neural connections. Finally, the conjecture is adopted that communicability can be used as a criterion of consciousness and free will is defined as a conscious version of relative freedom. The resulting notion is logically self-consistent and it describes an observable phenomenon that agrees with our experience.
\end{abstract}

Keywords Physics · Causality · Free will · Memory · Consciousness · Incomplete knowledge $\cdot$ Trial and error

\section{Introduction}

Juergen Ehlers knew about my attempts to understand free will from my talk in May 2007 for seminar Philosophical Problems of Physics at Charles University. He was visiting Jiří Bičák's relativity group and it was not sure that he would attend the talk.

\footnotetext{
P. Hájíček $(\bowtie)$

Institute for Theoretical Physics, University of Bern, Sidlerstrasse 5, 3012 Bern, Switzerland e-mail: hajicek@itp.unibe.ch
} 
But he did come, took a place in the first row, followed everything with interest and, as we all knew him, interrupted often with questions and comments. He understood perfectly what I was talking about and even seemed to like some statements. In one comment, he stressed importance of the ideas for teaching physics and science in general (which I have completely neglected). After the talk, we proceed with lively discussion for about one hour and he turned my attention to some literature, which would help me later. Juergen was the first of my colleagues who thought seriously and in some detail about my ideas, which constituted an unexpected encouragement. I was full of admiration for Juergen's scope and independence of judgement.

If anything happens, we have the tendency to ask two questions: 'What is the cause of it?' and 'What am I to do?' The questions originate in some deep (perhaps unconscious) convictions. The first is that every event must have a cause and the second that we can influence things by a suitably chosen action. In our practical life, the resulting two activities go hand in hand. For example, the knowledge of causes and effects improves our ability to choose actions so that they have the results we desire.

However, if we try to build a serious theory that would provide a more exact formulation of the convictions as well as underpin and explain them, we run into well-known difficulties.

To formulate the first conviction, most thinkers arrive at the principle (Plato, Timaeus):

Everything what happens must happen by a cause because it is impossible that anything comes into being without cause.

A more recent formulation is that by [12]:

An intellect which at any given moment knew all the forces that animate Nature and the mutual positions of the beings that comprise it, if this intellect were wast enough to submit its data to analysis, could condense into a single formula the movement of the greatest bodies of the universe and that of the lightest atom: for such intellect nothing would be uncertain; and the future just like the past would be present before its eyes.

This principle is called determinism; it gives a more exact formulation of the first conviction. The antithesis of determinism is that there are events that do not have causes. We shall call such events random. The antithesis has become popular in physics by the influence of quantum mechanics. A question is, how it can be made compatible with our tendency to look for causes.

A more detailed formulation of the second conviction and of the problem to understand it is the following [5]:

Any acceptable explication of free will:

1. must entail that free person could have chosen otherwise,

2. must explicate the control that free will requires,

3. must explicate the "sensibleness" or "rationality" that free will involves.

The first point assumes that there is a range of options from which one chooses. An important question is which conditions determine the range. An opinion that is often met can be characterized by adding the words 'all other conditions remaining 
the same' [22, p. 95] to the first point. More precisely, these conditions, which we denote by (A), can be formulated as follows

...given the total state of ourselves and the world at the time along with the laws of nature, it is open to us to do one thing next and open to us to do another instead.

\section{[16, p. 135].}

The 'control' mentioned by the second point requires that the choice from the range of options ought to be 'up to us'. An example of a theoretical idea that explains more precisely what this means is [16, (p. 211)] 'ultimate control over $x$-ing by $S$ ', where $S$ is an agent and $x$ is an action (even a mental one): A cause of $S$ 's $x$-ing at $t$ that includes no event or state internal to $S$ does not exist at any time before $t$. For example, the causal chain that ends in $x$-ing ought not to start before $S$ was born.

Determinism and its antithesis are examples of theoretical hypotheses while condition (A) and the ultimate control are theoretical notions. Theoretical hypotheses and notions are necessary if we are going to construct a theory that brings our experience in order and explains it. There is, however, one rather disturbing feature that the above hypotheses and notions have in common.

For example, determinism does not imply any specific observable effects. If we cannot identify a cause of any event, then there is always the excuse that the cause exists but is too elusive to be seen. Hence, determinism can be neither proved nor disproved by any observational data. ${ }^{1}$

Similarly conditions (A): How is an experimenter to recognize and describe, what the total state of the world is? How could he/she reconstruct it at some other time $t^{\prime}$ in order to make some results reproducible? This is likely to be impossible even in principle.

The hypotheses and notion with such a weak relations to experience are often called metaphysical. Moreover, it seems that the above metaphysical ideas can be identified as the source of the well-known difficulties. An example [11] is the following. Under conditions (A) determinism leaves only one option so that nobody could have chosen otherwise. The antithesis of determinism may allow for a non-trivial set of options under the same conditions but there is no control: nobody can influence random events.

We shall utilize what may be called the scientific methods (for more detail see [19]). One idea thereof is the primacy of observational data. Scientific notions ought to possess sufficiently strong observational aspects and scientific hypotheses ought to imply sufficiently specific observable consequences, the so-called predictions, which enable us to test them. Another requirement is the reproducibility of results so that they may be tested at different times and by different researchers. Thus, we must keep metaphysics in reasonable limits.

In the first part of the paper, we ask whether the science, in particular the physics, can make do without determinism and what notion could replace it. Moreover, we would like to see, whether the well-known scientific laws can admit any kind

\footnotetext{
1 Determinism is different from the hypothesis that each event from a specific class $C$ is a cause of an event from a specific class $E$. In fact, most scientific laws have such a form. They can be tested (but cannot be proven).
} 
of freedom. In Sect. 2, we find that even Newton mechanics allows some freedom because it cannot be reduced just to its dynamical equations. Only after a system and its initial state is chosen, the motion is determined uniquely by the equations. In quantum mechanics, a similar freedom of choice is augmented by the statistical character of the theory. This means that the dynamical laws do not determine the processes uniquely even if a system and its initial state are both fixed. We shall also see how random events and choices can be made compatible with (classical) general relativity. We introduce the important notion of uniqueness of history and show that space-times of general relativity can be interpreted as histories of completed time evolutions.

In Sect. 3, the resulting theory of causality is briefly described. The so-called event causality is shown to be sufficient for all purposes of physics. The time reversibility of dynamical equations is explained. This helps to understand the fact that the causality relation of two events is necessarily oriented and that there is no need to introduce new notions such as that of state causality. Following Hume, we distinguish the causation principle from the causality principle. We try to weaken the causation principle in agreement with the standard interpretation of quantum mechanics. The structure of the causality principle is found to involve a kind of conditions, which we shall call relevant conditions. The important feature of relevant conditions is that their occurrence can be recognized by observations. With each fixed set of relevant conditions, a definite option range is associated. Such a range represents a relative freedom, i.e., freedom with respect to the relevant conditions. Relative freedoms are observable phenomena and can be encountered in physics, biology and psychology.

Then we turn to biology in Sect. 4 and find that, in a unique and well-defined sense, living organisms are more flexible than certain class of automata. The flexibility is based on Popper's concept of trial-and-error processes (see [19, Chap. 6], On Clouds and Clocks). One of the fundamental features of living beings is the presence of some form of memory. We assume understanding of memory such as given in the book by Squire and Kandel [24]. An important fact about living beings is incomplete knowledge as represented in their memory. This leads to the existence of the so-called unforeseen events. Trial-and-error is the method by means of which living organisms cope with unforeseen events. We describe the ranges of options-mutations, motions and neural connections - that underlie choice of trials and we give the account of the corresponding relative freedoms.

Free will with the three properties listed above seems to be a natural part of this biological theory. Its only special feature is some conscious component. Section 5 conjectures that communicability can be used as a criterion of consciousness. This has some interesting consequences for the role of consciousness in free will. Relevant observations and experiments such as Libet's (2002) are re-interpreted accordingly.

A shortened version of the present paper is [9].

\section{Physics}

It may be surprising how much the study of physics and its methods will help us to understand and clarify the notion of freedom. 


\subsection{Newton mechanics}

We start the discussion with Newton mechanics and its structure. To describe the structure, a few abstract and general notions are needed: the system, the dynamical equation and the state.

The system in Newton mechanics consists of a given number of particles with given masses, on which given forces act. After a system is chosen the theory determines its dynamical equation. To obtain a unique motion, a state of the system must be chosen at a given instant of time, mostly at the beginning of the motion and then evolved by the dynamical equation. A state is determined if the coordinates and velocities of all particles are given.

The choices of a system and a state are not restricted by the theory. These freedoms are usually understood in a passive sense. For instance, the freedom of state is seen as the applicability of the dynamical equation to many different situations in which a system may be found in nature. Similarly for the freedom in the number of particles, their masses and the forces. It looks like a paradox but it is just logical: the more general a law is the more choice it admits.

Can we go a step further and interpret the choices of a system and a state as an active freedom of physicists? Such an assumption could be formulated as follows: Physicists are free to choose a system from a broad class of systems and a state of the system from a broad class of the system states. Then, they can construct this system in this state in a laboratory at an arbitrary time. However, the words 'physicists are free' have only a vague meaning here, they are expressing an impression rather than an established fact. We shall try to make it clearer in Sect. 3.

The impression seems to be always possessed by experimental physicists (disregard the possible lack of funds). In any case it is not only compatible with empirical praxis as well as with everyday laboratory work but the experimental freedom seems to be a tacit assumptions made by scientists generally. Mathematics and physics are often taught or understood as rigid and 'dead' sets of rules. This is not how these sciences are actually done!

If we assume that the world in its entirety can be reduced to a system of massive particles and forces between them so that all properties of all objects could be calculated from their mechanical parameters according to Newton mechanics then the future or past state of the world is uniquely determined by its state at the present time. This is the origin of Laplace's formulation of determinism.

According to our contemporary understanding, the assumption that the world can be described, in all its detail and in its entirety, by Newton mechanics is wrong. Thus, the theoretical support for determinism provided by it has broken down. Still, Newton mechanics is a good approximation in many cases and this makes it an alive and often used theory even today.

\subsection{Quantum mechanics}

Quantum mechanics has the same basic structure as Newton mechanics. Again, there is an affluence of various quantum systems. With each system a dynamical equation 
and a set of states is associated. Given a state at a time instant, then the state at any other time can be calculated from it by the dynamical equation and is unique.

The choice of system and state is not restricted by any rule in quantum mechanics either. The only difference is that the active version of it is explicitly formulated in some textbooks of quantum mechanics (see, e.g., [18, p. 50]).

The nature of the states of quantum systems is, however, very different from that of their Newton counterparts. They must enable a new feature, namely the statistical character of quantum mechanics. Given a fixed state of a system and a quantity that is measurable on the system, then its repeated measurements generally give different results even if both the state and the measurement are the same. Only the probability distribution of the results can be calculated from the state.

More precisely, a quantum experiment looks as follows. It consists of a number of individual measurements. In each measurement, we obtain a single quantum system (for example a photon) from a source (a laser, say), which is some macroscopic apparatus. The macroscopic structure of the source determines the quantum state of the system obtained from it uniquely. The (macroscopic) arrangement of the source and the measuring apparatus (for example a photographic plate), which is again a macroscopic system, is the same for each measurement. In each measurement, we obtain a certain value (for example a position) which can be read off at the measuring apparatus (for example, as a black point at the plate). If the experiment contains sufficiently many measurements then the frequencies of the values obtained are well approximated by the probabilities calculated from the state.

It is very important to notice that everything done by an experimenter is to manipulate and observe macroscopic devices. Quantum mechanics only identifies the cause of the distribution of the measured values: it is the choice of both a source and a measuring apparatus. But it does not specify any cause for a particular measurement giving this and not another value. As the values are visible by naked eye on a macroscopic body, it is the macroscopic behaviour that is not always predictable.

\subsection{General relativity}

Can the existence of random events and choices be included into a coherent picture of the whole world? In particular, is it compatible with the rest of physics? We have seen that Newton mechanics is only valid in a restricted domain so that its deterministic character does not prevent random events elsewhere.

However, there is another modern theory called general relativity. It describes the world on large scale. An important theoretical concept of general relativity is that of space-time: a four dimensional manifold carrying a Lorentzian metric and containing matter. The structure of general relativity is similar to that of Newton theory. It admits a number of different space-times, there are states forming certain sets that are different for different space-times and space-times must satisfy a dynamical equation.

The large scale character of general relativity is important if we are to compare it with Newton or quantum mechanics. There does not seem to be much choice of a system if the theory intends to describe the whole universe. Moreover, we cannot 
require from physicists to set up an arbitrary state of the world in their laboratory at an arbitrary instant of time. However, we can say that different space-times represent different models of the universe.

The next problem is that there is no mark on a space-time that would distinguish the present instant and there is no difference between the general structures of past and future (this is similar to Newton mechanics). The usual interpretation is to say that this difference is purely subjective and that the present instant may be anywhere depending on where observers happens to be. The space-time is considered as an observer-independent description of the macroscopic world for all times.

However, the picture of time that follows from the existence of random events is dominated by an asymmetry between future and past. The future does not yet exist and more possibilities are open to it. The past is fixed because the choice of the possibilities is done only at present instants of time. Such an asymmetry is not new in philosophy, see, e.g., [20].

Consider the past. It can exist only as a memory or another kind of record that an observer, or a family of observers, can make about the observations done within each of their progressing presences. Only in this indirect way does the past have to do with reality. The past as a (processed) record is in principle fixed in all aspects and details. There are two very different reasons for that. First, the choice between free alternatives has been done and no change is possible any more. Second, we usually suppose that different observations or observations by different observers concerning the choices can finally be put into an agreement. This is a rather non trivial hypothesis on which, in fact, all of the science is based. We call this hypothesis uniqueness of history. It has a natural explanation in the philosophical realism [21]; for a more contemporary discussion of realism, see [23] analysis in Chap. 10.

As for the future, its very existence is a hypothesis based on the analysis and extrapolation of the past. Similarly, we can extrapolate the existence of various structures and the validity of laws found in the past. Since there are also unpredictable aspects, one can say that some part of the world is newly created ([19]: 'chosen by nature') at each present instant of time, another part is determined by the past.

Now, we can give the answer to the question of how space-times of general relativity are to be interpreted: Any space-time is just a hypothetical past of a completed time evolution [8]. (A space-time can be viewed as one possibility for a complete evolution of universe including definite random choices made at each time). The problem of asymmetry between future and past then does not arise because we are working only with pasts. If we accept this change in the interpretation of general relativity, then the existence of random events becomes compatible with the whole of the contemporary physics.

\section{Causality}

\subsection{Event causality}

Causality and some related notions are very important in discussions about freedom. In this section, a theory motivated by physics is described. It also defines our dictionary 
and tries to prevent confusion caused by the fact that different texts use the same words in different meanings.

Let us start by stipulating that causality concerns primarily relations between events. An event will be understood as what happens at roughly one point and at one time (more precisely, in a small space-time neighbourhood). Thus, an event is not just a small space-time neighbourhood but something must also happen there. This kind of causality is often called event causality. Other kinds of causality can be found in literature. For example, the structure of a system is considered as the cause of its properties. It is not clear in how much this has to do with a logical reason rather than with a physical cause. In any case, such important relations can be accounted for under the heading of models and theories, not under causality, and so they will not be lost. Another example of causal relation that is not a relation between events is the so-called 'agent causation' [11] not need it.

Event causality is sometimes criticized (e.g., [3, p. 66]) as a naive, pre-scientific kind of causality and the causality which is met in the modern field theories is described in a different way: the state of a field at an instant of time is the cause of another state at a later, or even earlier, time instant (state causality). Of course, the state of a field at a time is not an event but we can consider such a state as a set of events. ${ }^{2}$ Indeed, to describe such a state, the values of some measurable quantities must be given at all points of space at a given time, and the fact that these values occur at various space-time points is nothing but a set of events in our sense.

The time reversibility of some dynamical equations is also considered as a difficulty for event causality, which distinguishes cause from effect and is, in this sense, oriented. A short reflection however shows that the reversibility of dynamical equations does not mean the reversibility of the relation of cause and effect even for state causality. It is true that we can often reconstruct the state at an earlier moment if we know the state at a later one. But this is a logical reconstruction. Indeed, we cannot change the state in the past by manipulating the state at the present. The re-constructibility may be interesting in that it can be considered as a criterion of completeness of the event sets which one has to do with. Complete means here that the set is equivalent to a state, because else the states at other instants of time would not be uniquely determined.

Further discussion of the reversibility needs a clarification of the meaning of this concept first. Indeed, it definitely does not mean that the time flow can be inverted. At most, the order of some aspects of a possible motion can be inverted so that another possible motion results. For example, in Newton mechanics, the coordinates as functions of time $t$ describe a trajectory of a motion. One can substitute $-t$ for $t$ into the functions and another possible trajectory will result (if the forces are time-reversal invariant). Now, the states of Newton mechanics are completely determined only if both coordinates and velocities are given. But the velocities at the corresponding points after time reversal are not the same as before, they must also be inverted. Hence, the same states are not running in the reverse order if we 'reverse time' and the final state of the original motion cannot be the cause of its initial state. To summarize, it seems that some version of event causality is an adequate notion, and it is even more

\footnotetext{
${ }^{2}$ Even in quantum mechanics, any specific preparation of a state can be considered as a set of (macroscopic) events.
} 
satisfactory for physics than various reversible state causalities. Let us elaborate on the corresponding causal relation in more detail.

A causal relation is an oriented relation between a set of events $\mathcal{A}$ called the cause, and an event $B$, called the effect. If $\mathcal{A}$ occurs, $B$ must also do ( $\mathcal{A}$ is called by some philosophers 'sufficient cause'). For example, in Newton mechanics, the elements of a cause $\mathcal{A}$ can be values $x_{n}^{i}$ of the three coordinates and velocities $\dot{x}_{n}^{i}, i=1,2,3$, for all particles $n=1,2, \ldots, N$ at some time $t$. Then, $B$ can be certain pair $x_{n}^{i}, \dot{x}_{n}^{i}$ concerning $n$th particle and $i$ th coordinate axis at a time $t^{\prime}$ larger than $t$. If we take all such $B$ 's at the time $t^{\prime}$, then we have a complete system of effects so that we can reconstruct $\mathcal{A}$. This suggests that we can collect several effects of the same cause in a set and call this set also an effect of the cause. But, unlike causes, the composed effects seem to be always decomposable into their event elements.

Locality means in physics that causes always precede their effects in time, $t(\mathcal{A})<$ $t(B)$. In modern physics, this mostly implies that the effect must lie in the future light cone of the cause: causing does not travel with a velocity larger than the speed of the light. There are some exceptions, but these are always due to some special circumstances that can be detected. For example, in the case of super-luminal group velocities in some media etc., see [13], there always seems to be an inertial frame where no effects precede their causes, such as the rest frame of the medium.

\subsection{Principle of causality}

Thinking about causality was strongly influenced by [10]. Hume distinguished two ideas within the notion of causallity. They can be called [23, p. 138] principle of causality, which states that 'like objects, plac'd in like circumstances, will always produce like effects' ([10], p. 105), and principle of causation, which maintains that 'whatever begins to exist, must have a cause of existence' ([10], p. 78). ${ }^{3}$ Hume also listed all properties of the causal relation given above.

The principle of causality seems to be the most important part of the theory because without it the observation of causal phenomena would be impossible. Let us try to give the principle a more precise form. First, we say 'equivalent' instead of 'like': equivalent causes and equivalent effects form classes that are determined by some conditions.

Physics and its ways can help to understand the conditions. First, the objects which physicists work with are called systems. Systems usually come in many copies and there is some way to recognize that two systems are equivalent.

Second, physicists study systems under specific conditions that must be reproducible (in a laboratory, say) or at least recognizable and often encountered (in astrophysics, say). The conditions should not in general contain the time and the position. This enables us to observe equivalent causes at different times and positions and to check that there is a pattern. That is, observations can be repeated in order to confirm or extend previous results. We also assume that every occurrence of such conditions consists of a set of events. The conditions do not have any special name in physics, but

\footnotetext{
3 Hume himself criticised the idea of causality, but this has no direct importance for our theory.
} 
for our purposes it is advantageous to give them one. Let this be relevant conditions because they are indeed relevant for the study or the experiment.

In fact, the notion of relevant conditions is the basic tool of our understanding the world around us. All laws of nature have been discovered by looking what happens if some relevant conditions occur repeatedly. In this way, each particular causal relation becomes an instantiation of a general law of nature. ${ }^{4}$

The science makes a heavy use of relevant conditions for example in its experimental methods. The experiments are to be reproducible and so their conditions have to be defined carefully. It seems, however, that the recognizability and reproducibility of the conditions is never absolute; there is no absolutely clean experiment. There always seem to be uncontrolled influences; in any case, repeated experiments always give some dispersion of numerical values. The lack of an absolute control about the purity of the conditions can be quantified by the dispersion (mean squared deviation). In practice, one views a control as excellent if the dispersion is sufficiently small.

\subsection{Principle of causation}

Let us turn to the principle of causation. Historically, this is a very old principle. For example, almost the same words were used by Hume and Plato (see the Introduction). From the principle of causation, it follows that we could predict everything that happens in the future of a time instant, if we knew all kinds of causes and if we knew everything that happened in the past of the time-determinism. Determinism can also be described as follows: Given the complete state of the whole universe at a time instant $t$, then there is only one possible way of how the universe evolves in the future or past of $t$.

It has been explained in the Introduction that the principle of causation and the associated determinism cannot be proved or disproved. What we can control in physical experiments with, and in observations of, a system is always only a tiny part of the universe so that we can recognize if it satisfies some relevant conditions. A system subjected to given relevant conditions exhibits a behaviour. If the conditions occur again, the system can show the same or a different behaviour. In this way, one can find a number of alternative possibilities that are open to the system under the given conditions. If, after many repetitions, no new possibilities occur we can assume, that they have all been found. Such a complete system of possibilities is called option range. The existence of the options can, therefore, be experimentally tested. The knowledge about specific option ranges form an important part of science.

Most contemporary physicists simply assume what we call the Weak Causation Principle (WCP) and what we can formulate as follows: Every event is an element of an option range determined by some maximal relevant conditions.

Relevant conditions are called maximal if adding any further condition does not decrease the number of options. If the corresponding option range contains only

\footnotetext{
4 Causal relations and laws of nature cannot be proved by pure logic from pure observational evidence. This was also shown by Hume. We can agree with Hume by saying that they remain only more or less probable hypotheses, but we still maintain that this does not make them useless.
} 
one element we have a causal relation of the usual type. But WCP admits also cases when the range contains more alternative possibilities. The existence of such cases is assumed by the standard interpretation of quantum mechanics. WCP does not comprise much more than just what experience tells us but it is sufficiently strong to explain our search for causes, interesting relevant conditions and option ranges.

After about 80 years of quantum mechanics, WCP seems to be a simpler and more comfortable hypothesis than the determinism. To introduce additional causes (in quantum mechanics, the so-called hidden variables) into the theory makes it technically more complicated. Moreover, in this way, something is introduced that does not have even an indirect relation to what can be observed today. By Occam's razor, we are better to abstain. If evidence will emerge sometimes in the future that will have any relevance to the problem, it will most likely be very different from anything what we could imagine in our wildest dreams today. However, it is not true that quantum mechanics has proven determinism to be wrong.

\subsection{The notion of relative freedom}

The uniqueness of history makes determinism impossible to disprove and freedom difficult to see. Still, physical experiments show the way of how a rational notion of option ranges can be introduced. Crucial is the concept of relevant conditions. They are weaker than conditions (A) mentioned in the Introduction and posses a closer relation to observational data.

We can define: If a system has an option range that contains more than one element under some fixed relevant conditions, then we say that it has a freedom relative to the conditions, a relative freedom. Hence, one and the same system can have several different relative freedoms depending on the choice of the relevant conditions.

Our definition of option ranges does not mention the way in which its alternative possibilities are realized. It can be an effect of a cause that lies outside the relevant conditions (then the conditions are not maximal) or it need not have any cause. The way of the realization of a fixed option may even differ in different cases of its realization. The existence of relative freedoms itself contradicts neither the existence of causes nor that of random events.

Let us stress that relevant conditions may define an important and useful relative freedom even if they are not maximal. An example is connected with the so-called emergent phenomena. These are properties of complex systems that cannot be derived exclusively from the properties of its individual constituents. The possibilities of how given constituents can combine form an option range. Consider for instance electrons, protons and neutrons. They can combine into about one hundred stable atoms that in turn can combine into zillions of stable molecules, crystals and mixtures. This is a huge option range, which underlies the surprising wealth of structure in nature.

A noteworthy example of non-maximal relevant conditions is the relative freedom that is associated with each scientific law such as dynamical equations in physics. Indeed, the more general a law is, the larger the number of individual cases can be ruled by it. If we define the relevant conditions as the applicability of the law, then 
the individual cases form the option range of a relative freedom. The ability to use the law entails a deep knowledge of this freedom.

We now have a better understanding of the impression about the freedom of physicists referred to in the subsections on Newton and quantum mechanics. There are relevant conditions: to have a well-equipped physical laboratory, full stop. There is an option range: all mechanical or quantum mechanical systems that can be constructed in the laboratory and all states, in which the systems can be created. First, it is clear that the statement can be given an arbitrarily rigorous form, second, it expresses exactly the freedom that is necessary to do physics and, finally, it seems that it is an adequate explanation of the freedom impression of the physicists.

\section{Biology}

Let us define the living organisms provisionally by counting, just taking over the existing listing and taxonomy. Experience shows that living beings all have what we can call elementary needs: they feed, they reproduce and they avert or avoid danger (we understand these words in a sufficiently general sense so that they apply to all living organisms). Then, their behaviour can be understood as ultimately motivated by the elementary needs. There is therefore what can be called teleonomy. The teleonomy is 'built in the genes'. An account of it is given by [17].

Of course, there are organisms that have much more sophisticated motivations. It seems, however, that the 'higher' motivations can be understood as derived needs, needs that have their origin in strategies serving to satisfy the elementary needs. For example, some animal species are gregarious, and it is more or less clear, how a suitable way of life in a group makes feeding, reproduction and averting dangers easier or even possible in a given niche. Then, seeking, gaining and keeping a suitable position in a group may be understood as a derived need.

Another important property of the known living organisms is that they are more flexible than the machines that follow fixed algorithms. A generally used mathematical model of such a machine is the so-called [25] machine. From the biological point of view we can describe an algorithm as follows: it determines specific responses to specific stimuli and there is a fixed list of the stimuli (a list of the responses is determined by the organism). ${ }^{5}$ In our language of conditions and options, we can say that there are conditions - identified with always one specific stimulus of the list - that possess only one option, a response from the other list. To be sure, the behaviour of all living organisms including people follows in some stages some such program. Let us call it routine. However, the strategies of living species are not exhausted by routines. On the other hand, we do not claim that it is impossible to construct a machine that is as flexible as a living organism. Observe that the lists of stimuli and reactions represent information, e.g., some knowledge about how the stimuli are related to the elementary needs.

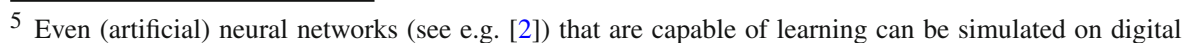
computers, i.e., in principle on Turing machines. The reason is that they can only learn to associate a response from a fixed closed list with a stimulus from another fixed closed list.
} 
Suppose that an event occurs that is not in the list of stimuli, but it none the less still has relation to the elementary needs. Let us call such an event unforeseen. We leave open whether an unforeseen event has had a cause or not. The existence of unforeseen events can be interpreted as an instance of incomplete knowledge: the knowledge is missing that concerns both the link of the unforeseen event to the elementary needs and the way in which this link can be used for a suitable reaction. The memory of an organism does not contain any direct representation of such knowledge.

The flexibility means that all species are equipped with facilities (body structures) that enable them to reprogram the routines if some unforeseen events occur. An important property of unforeseen events is that there does not exist any list of them. Hence, a solution to such a problem cannot be programmed in the way Turing machines work. A general procedure serving this purpose that can be observed in all living beings has been highlighted by Popper [19], who has called it the 'trial-and-error method'. The same principles have been applied by Edelman [6] just to the way brains are working. However, his 'neural Darwinism' is aimed at perceptual categorization, generalization and memory rather than at free will.

In our language of conditions and options, we can give the following account of the 'method'. Consider an organism such as a mouse and let some specific conditions contain an unforeseen event and let the conditions allow some range of options for the mouse response. Then, the mouse that is confronted with the conditions must be able to make choices from the range. Let us call the procedure of such choice realization. The realization process may be random or causal or something in between-this is irrelevant for our theory. The ability to perform the realization process needs freedom that is possessed by all living beings. Pointedly said, it is the liberty to err. After each choice, the mouse perceives what happens. Often, the result is negative (error). The next time when the conditions occur, the mouse makes another choice. And so on, until the result is positive. The good choice may then be remembered and the routine thus reprogrammed. This part of the procedure is called selection.

It is clear that a necessary condition for the procedure of trial and error to work is some kind of memory. The errors have to be remembered so that they will not be repeated. The selections have to be remembered, in order that they will be chosen. And indeed, some kind of memory is the most important part of any living being.

Our theory of biological freedom explains the frequent observation that structures evolved to serve certain needs are subsequently used for very different puposes. Let us now look to see how it works in more detail.

\subsection{Mutations}

The most basic kind of memory in biology is an inheritance molecule such as the deoxyribonucleic acid (DNA) and the basic option range in biology is formed by mutations. Its alternative possibilities are the changes in the DNA. Mutations come about in a realization process that may work all the time (as, e.g., for bacteria) or during specific periods (meiosis for sexually reproducing organisms; a generally understandable description thereof is [4]) and it seems to be random. 
If the option range is wide enough then some of its possibilities are advantageous for the mutated organism in the sense that they lead to the proliferation of individuals with this mutations and to relative suppression of the others. In this way, the selection process works. As a rule, the selection is not random and causes can be found for most selections.

Observe that the unforeseen event can be answered only if it comes after the suitable mutation is established and remembered. For example, if a new antibiotic is applied to a bacterial infection, there must already be bacteria with a suitable mutation. Thus, in the special case of mutations, the unforeseen events themselves take part in the selection process.

\subsection{Neuron connections}

Some multicellular organisms such as animals possess an option range with alternative possibilities called motions. Parts of the animal's body, e.g., trunks, legs or eyes, can take different relative positions without inhibiting other functions of the body. The relevant conditions are simply the internal anatomy and physiology of the body as well as the external circumstances that allow changes of such positions.

Animal motions are usually organized with the help of the nervous system. Properties of nervous systems are important for us but a simplified picture that follows will be sufficient. Much more detailed neurology supporting our approach can be found in [6] 'theory of neural groups selection'.

Experiments show that certain nerve signals trigger certain motions. Sequences of motions such as running or flying are brought about by specialized sets of nerves connected in a particular way. Influence of sensory data on motions is made possible by connections of other sets of nerves. The connections of neurons can thus code for processes containing motions and can themselves be considered as a memory. Some neural structures are inherited: the connections are built up according to the DNA blueprint. This kind of memory serves for routines and cannot be used for trial-and-error processes. The connections have been selected by the process based on the mutations and the contents of the neural memory is equivalent to a part of the DNA.

Other connections of neurons can, however, be altered also during the life of an individual organism. Let us consider changes enabled by the so-called synapses. The alternative possibilities of how the strengths of synapses can be chosen form an important option range for animals. The relevant conditions here are the positions and variation range of all flexible synapses. The simplest example of such an option range and a memory based on it can be recognized in the learning by invertebrates as described by Squire and Kandel [24]. There are three kinds of such learning: habituation, sensitization and classical conditioning. Let us look at the habituation. The other two kinds of simple learning are of similar nature.

Let some new stimulus that does not carry any danger by itself cause some kind of alarm response by an animal. In this case, we can arrange a training session in which this stimulus is occurring more often without being followed by anything harmful. Then, the response becomes gradually weaker until it practically disappears. This is 
first remembered only for a short time of, say, ten minutes. If the training sessions are repeated sufficiently many times over a sufficiently long time, then the new reaction can be remembered for a longer time, such as several weeks.

The connectivity of the neurons as well as the physical and chemical processes in the synapses that are necessary for such learning are well understood. We can describe the learning in our language in agreement with these neurological details. The relevant conditions are the occurrence of the stimulus and no harmful event following it (the unforeseen event). The options are the degrees of alarm. The realization process is carried out by the chemistry of the synapses and is rather systematic: the degree of alarm is approximately decreasing. Observe the important role of the repeated occurrence of relevant conditions so that an animal can perceive what its trials are leading to. The no-alarm option is selected after a chain of sensory inputs. What has been learnt is a specific response to a specific stimulus. This is not just a kind of 'second-order' Turing machine: true, a response to a stimulus chain is a change of a response to a stimulus. But, as it was already stressed, there is no list of unforeseen events. The method is trial-and-error.

More advanced kind of learning and trial-and-error processes are enabled by the so-called declarative memory. It needs a collaboration of two complex nervous structures: the cortex and the hippocampus. Consider for example the experiments with rats in the Morris water maze [24]. This is a large circular pool with murky water and steep walls that cannot be climbed by the animal. Under the water not at the centre of the pool, a slightly submerged platform is hidden.

The rats are put into the water at some fixed position in the pool. The subsequent chaotic motion of a rat in searching for something (it does not know about the platform) has the character of a trial-and-error process. The options are nervous signals that code for and trigger different possible swimming motions. We do not need to know in detail the nature of these signals and simply call them portable neural representations (PNR). They seem to occur in a rat's brain in a more or less random way. Some of them may be related to some stimulus from outside as well as from the rat's memory. The realized PNR's that lead to motions are the trials of the process. Finally, the successful PNR representing a more or less direct swim to the platform is selected and remembered.

After this training, the experiment is modified in order to see what kind of knowledge has been acquired about the location of the platform. There are two groups of rats: those with healthy hippocampus and those with lesions there. The rats of both groups are able to swim directly to the platform from the position where they have been always put into the water during the training. Next, the rats have to start from another position in the pool. It turns out that the normal rats swim to the platform from any position without searching while those with lesions have to fall back on trial and error.

To explain these observations, one possible assumption is that the healthy rats acquire more knowledge about the layout of the room than the others. The knowledge is in the form of a PNR that somehow represents the layout. It is possible in principle because the room with the pool is not rotationally symmetric: its walls carry some cues. The layout PNR can be used by the rats to work out the suitable motion from the sensory data on where they have been put into the water. If this calculation takes the 
form of a purely mental trial-and-error process see [6], then the new starting position $i s$ an unforeseen event and what the healthy rat does is not a routine. If not, then the healthy rats have simply learned a better kind of routine than the others.

Another hypothesis is that the acquired layout PNR is similar for both groups but the rats with lesions cannot work out from it the correct motion. There are two possibilities. First, the purely mental trial-and-error process cannot be performed without hippocampus. Second, the routine calculation cannot be done so. In this case, the lesser calculation ability has the same effect as the incomplete knowledge.

By the way, we observe that none of the rats chooses to swim merrily around instead of searching for something. They apparently have an inbuilt dislike for staying in water that has some connection to their elementary needs.

\section{Freedom of will}

The freedom of will is usually understood as the ability to choose consciously an idea of an action and then carry out the action accordingly. The general notion also agrees with free will having the three properties listed by Double (see the Introduction). In this section, we try to construct a theory explaining these points.

Clearly, the choice is only possible if there is an option range corresponding to some conditions. Our main idea is to replace conditions (A) by the relevant conditions. In this way, our theory of free will becomes a part of the general biological theory described in the previous section. The only new feature that makes a relative freedom to free will is a suitable conscious component.

As for ideas, we assume that each is associated with a portable neural representative. $^{6}$

The nature and role of consciousness and how the consciousness is represented by neural processes in the brain is not known. However, some incomplete and phenomenological understanding might be sufficient for our purposes. In particular, an important criterion of consciousness is the communicability: a conscious idea, or that an idea is conscious, can be told to others. In fact, this criterion is crucial for the experimental work done e.g. by Libet: there is no way experimentalists can be informed on consciousness of another person independent of what the person tells them.

There are various phenomenological hypotheses on consciousness. Some surprising observational data see [26] suggest (but do not prove) the conjecture that consciousness is only an epiphenomenon. If this were true, then the free will in our sense would still exist: we could define it as conscious experience of relative freedoms. However, it seems that the data admit a stronger conjecture.

If we adopt the communicability criterion, then it follows that consciousness helps us actively to carry out complex mental work. A necessary component of such a work is a formulation of partial results from time to time in a way that is understandable to others even if no communication is planned. This must be conscious according to

\footnotetext{
6 The ideas are mental states or processes and PNR are neural states and processes. We accept the solution of the mind body problem as described in detail by Searle [23]. Roughly, the ideas and PNR are different entities, which, however, can be considered as two sides of the same thing. Similar thoughts can be found already in [7] and perhaps even before Fechner.
} 
the criterion. The purpose is to check that nothing has been forgotten and that the whole is logically coherent. We can thus try the more general conjecture that consciousness provides active and manifold help with thinking. It is also supported by the observation that consciousness is always experienced in non trivial processes of thinking. Moreover, it explains why brains with consciousness have advantage over those without and why they, therefore, would evolve at all.

As free will is concerned, the conscious component in realizing and selecting ideas carries with it both advantages and disadvantages. On the one hand it helps thinking, on the other, it consumes more energy and time than unconscious processes. Indeed, the relatively long time needed for conscious processes is an established observational result $[14,26]$. Brain processes consume glucose at a pretty high rate. This suggests that there is also need for more energy. If conscious processes in the brain run parallel to unconscious ones or if they entail synchronous actions of many neurons [15], then they are expensive.

Our experience forces us to admit that unconscious processes take also part in thinking. What is their role? Let us look more closely at the experiments supporting the conjecture that actions are chosen unconsciously and that consciousness is only an accompanying phenomenon. A conspicuous feature of the experiments is that the actions asked for do not require any complex mental work. For example Libet asks for the choice of an arbitrary time instant for a simple motion of a wrist.

Hence, a simple alternative interpretation of such experiments is as follows. When the experiment is running, the unconscious brain does not switch on the consciousness at once because no complicated mental work is needed. It does the choice itself, only then 'drops a notice' to the consciousness to enable a possible veto and to the declarative memory to save it for later, maybe conscious, use.

Let us instead look at the game of chess. This is an activity that clearly needs non trivial thinking. First, a chess player has motives and desires to play or not to play a game, to win or not to win as well as some more special tastes of how to play. Some vague ideas about connections of these thoughts to the elementary needs could be imagined, but we leave the question open. In any case, we do not require the origin of the desires to be always a conscious, rational reasoning.

After the decision to play and win and after the start of a game, there are well defined option ranges: all moves that are allowed by the rules of chess in a given position. The moves are understood as ideas. Some positions with which a player is confronted during the game are unforeseen events as defined at the beginning of Sect. 4.

No chess player is able to calculate the game to the end from most positions; not even the fastest computer can do this in a reasonable time. The players are looking for a 'strong' move that is enabled by some properties of the position. If they become aware of any such move they calculate the possible consequences of it to the depth of several moves to check if the idea works. In this way, they learn more about the position so that after rejecting one idea, they are likely to get another, etc. It is not allowed to make trial moves with the pieces, hence we have a purely mental method of trial and error. Conscious processing is influenced by input coming from the unconscious brain. The players become aware of relations to other games that they played or studied in the past. The process of selection from trial moves is an example of process in which the help of consciousness can be experienced at many intermediate steps. 
The final point is that after having calculated the moves and selected among them with the help of consciousness the act of actually moving a piece may again contain unconscious elements. Indeed, it is often done in an utterly mechanical way that is not even remembered. The question whether the conscious mind directly moves the hand seems to be less important than whether the hand moves in accordance with the mind plan.

The chess example makes an important point. Consciousness is considered as a tool helping to choose from option ranges in complicated cases. The manner of its working as such a tool explains the third point by Double (see the Introduction): some rational and sensible aspects of free will. Indeed, as we have argued, one of the tasks of consciousness is to check that the selection is based on a logically coherent system of ideas and that it satisfies the desires.

A still stronger conjecture on the role of consciousness is a hypothesis that I would call the consciousness domination. The idea is that, on the one hand, desires and motivations are formed rationally in a conscious way and, on the other, that conscious thoughts can be direct causes for actions. Wegner [26] had thoroughly discussed the second part of this hypothesis, which he called the conscious will. He had drawn on a wealth of experimental and observational data and concluded that this kind of conscious will is an illusion. Now, the analysis of the chess game shows that the theory of free will explained in the present paper does not make use of any part of the consciousness-domination hypothesis.

Let us turn to the second point by Double. A scientific theory of control can go roughly along the following lines. It starts at the observation that all living organisms are born with some teleology: there are certain elementary needs that have to be satisfied. Humans are gregarious animals, with an involved hierarchical social structure. They need to find, gain and keep a suitable position in society as was mentioned at the beginning of Sect. 4. The needs are clearly given to us from nature and are not up to us. But the inbuilt teleology shows only general directions. In fact, we are overwhelmed by the huge manifold of choices to which different people are stimulated by their inherited teleology. Let us call the basic choices of this kind the options of self.

On the one hand, the choices from the options of self are not completely up to us. Clearly, some of them develop even without our conscious acting through breeding, education, accidental circumstances, etc. On the other hand, the corresponding desires and motivations need not even be compatible with each other and one has to deliberate and choose. Moreover, many of us have the experience of, as it were, consciously and freely changing a quite essential part of the self after some very important unforeseen event happens, although such events are rather rare. The choice must be done in agreement with our feeling of what is the best for us and this is the basis of some control. Next, a more or less complete, definite and constant self does not by itself determine uniquely our specific everyday choices. Again, there can be conflicting desires on the one and different ways of satisfying one desire on the other hand. The control means that we make our choices in the best possible accord with our self if an unforeseen event occurs.

If these ideas on control are properly interpreted then they can be recognized as a part of the teaching on the self in the contemporary psychology, see e.g. [1]. The difference between the present paper and the work of psychologists is that the research 
in psychology focuses on patterns of experience and studies the behaviour that is a consequence of our impression of being free to choose. Hence, a lot of useful work can be done without addressing the philosophical problem associated with a real free choice. The result is a purely phenomenological theory that does not explain its basic notions. Some do just that (Baumeister), others interpret the impression of free choices as an illusion (Wegner).

Our biological definition of free will makes it a phenomenon that is compatible with all scientific evidence and can be studied by natural sciences on the one hand and that seems to agree with everyday impression of the freedom as we all know it on the other. However, only some rough general idea has been be given here and many details still remain to be elaborated on.

Acknowledgments The author is indebted to Gilberto Collangelo, Victor Gorge, Claus Kiefer, Karel Kuchař, Martin Pokorný, Oldřich Semerák and Uve-Jens Wiese for reading some of earlier version of the manuscript and for suggesting improvements. Thanks go to the Institute of Theoretical Physics, Faculty of Mathematics and Physics of the Charles University, Prague for hospitality and discussion.

\section{References}

1. Baumeister, R.F.: The self. In: Gilbert, D.T., Fiske, S.T., Lindzey, G. (eds.) Handbook of Social Psychology, 4th edn., vol. 740, pp. 680-740. McGraw-Hill, Boston (1998)

2. Churchland, P.M.: The Engine of Reason, the Seat of the Soul. Mass: MIT Press, Cambridge (1995)

3. Campbell, N.R.: Foundations of Science. The Philosophy of Theory and Experiment. Dover Publications, New York (1957)

4. Dawkins, R.: The Selfish Gene. Oxford University Press, New York (1989)

5. Double, R.: The Non-Reality of Free Will. Oxford University Press, New York (1991)

6. Edelman, G.M.: Neural Darwinism the Theory of Neuronal Group Selection. BasicBooks, New York (1987)

7. Fechner, G.T.: Elements of Psychophysics. Holt, Rinehart and Winston, New York, 1966 (1860)

8. Ellis, G.F.R.: Physics in the Real Universe: Time and Spacetime. Arxiv, gr-qc/0605049 (2006)

9. Hájíček: Free will as relative freedom with conscious component. Conscious. Cognit. 18, 103-109 (2009)

10. Hume, D.: Treatise of human nature. Amherst: Prometheus Books. Book I, Part III (1992)

11. Kane, R.: A Contemporary Introduction to Free Will. Oxford University Press, New York (2005)

12. Laplace, P.: Théorie Analytique des Probabilités. Courcier Imprimeurs, Paris (1820)

13. Liberati, S., Sonego, S., Visser, M.: Faster-than-c signals, special relativity and causality. Ann. Phys. 298, 167-185 (2002)

14. Libet, B.: Mind Time. The Temporal Factor of Consciousness. Harvard University Press, Cambridge (Massachusetts) (2004)

15. Llinás, R., Ribary, U.: Proc. Nat. Acad. Sci. (USA) 90, 356 (1993)

16. Mele, A.R.: Autonomous Agents. From Self-Control to Autonomy. Oxford University Press, New York (2001)

17. Monod, J.: Le hasard et la nécessité. Edition du Seuil, Paris (1970)

18. Peres, A.: Quantum Theory: Concepts and Methods. Kluver, Dordrecht (1995)

19. Popper, K.R.: Objective Knowledge. Clarendon Press, Oxford (1972)

20. Popper, K.R.: Unended Quest an Intellectual Biography (Chapt 28 Meeting Albert Einstein). Fontana and Collins, London (1974)

21. Russell, B.: The Problems of Philosophy. Oxford University Press, Oxford (1959)

22. Searle, J.R.: Minds Brains and Science. Harvard University Press, Cambridge (Massachusetts) (1984)

23. Searle, J.R.: Mind a Brief Introduction. Oxford University Press, New York (2004)

24. Squire, L.R., Kandel, E.R.: Memory. From Mind to Molecules. New York, Scientific American Library (1999)

25. Turing, A.M.: Proc. Lond. Math. Soc. (ser. 2) 42, 230 (1937); [correction 43, 544]

26. Wegner, D.M.: The Illusion of Conscious Will. Mass: MIT Press, Cambridge (2002) 